\title{
Study on an Optimal Control Method for Energy Injection Resonant AC/AC High Frequency Converters
}

\author{
Yu-Gang Su ${ }^{*}$, Xin Dai ${ }^{\dagger}$, Zhi-Hui Wang ${ }^{*}$, Chun-Sen Tang ${ }^{*}$, and Yue Sun ${ }^{*}$ \\ $\dagger^{*}$ College of Automation, Chongqing University, Chongqing, China
}

\begin{abstract}
In energy injection resonant $\mathrm{AC}-\mathrm{AC}$ converters, due to the low frequency effect of the $\mathrm{AC}$ input envelope and the low energy injection losses requirement, the constant and steady control of the high frequency $\mathrm{AC}$ output envelope is still a problem that has not been solved very well. With the aid of system modeling, this paper analyzes the mechanism of the envelope pit on the resonant AC current. The computing methods for the critical damping point, the falling time and the bottom value of the envelope pit are presented as well. Furthermore, this paper concludes the stability precondition of the system AC output. Accordingly, an optimal control method for the AC output envelope is put forward based on the envelope prediction model. This control method can predict system responses dynamically under different series of control decisions. In addition, this control method can select best series of control decisions to make the AC output envelope stable and constant. Simulation and experimental results for a contactless power transfer system verify the control method.
\end{abstract}

Key words: Energy injection, Envelope control, Optimal control

\section{INTRODUCTION}

At present, there are numerous methods to realize AC-AC transformation in power electronics converters. The cyclo-converter is a typical method to realize the conversion from high to low frequencies. Matrix converters [1]-[3] and AC-link converters [4]-[7] are usually utilized to realize AC-AC conversions from low to high frequencies. However, their topologies are relatively complex. The energy injection resonant $\mathrm{AC}-\mathrm{AC}$ converter is a novel direct $\mathrm{AC}-\mathrm{AC}$ converter. This kind of converter can realize direct AC-AC conversion from a low frequency $(50 \mathrm{~Hz})$ to a high frequency $(20-100 \mathrm{KHz})$. The topology of the direct AC-AC converter is simple and easy to control. Thus this direct converter has been widely used in contactless power transfer (CPT) systems [8]-[10] and inductive heating systems [11]-[13].

However, there are two problems which make the constant control of the output resonant current difficult. They are listed as follows:

(1) The low frequency AC power source directly influences

Manuscript received Aug. 14, 2012; revised Dec. 29, 2012

Recommended for publication by Associate Editor Bor-Ren Lin.

${ }^{\dagger}$ Corresponding Author: toybear@vip.sina.com

Tel: +86-23-65112750, Fax: +86-23-65111221, Chongqing University

${ }^{*}$ College of Automation., Chongqing University, China the envelope of the output resonant current. There is an obvious current pit on the current envelope corresponding to the zero crossing region of the low frequency AC input. The envelope pit greatly influences the stability of the power transfer and produces a low frequency ripple which makes the output filter design difficult.

(2) The direct AC-AC converter is a typical dissipation oscillation system. The stability of the output current is greatly reliant on the control strategy between the energy injection and the freerunning modes. Moreovrer, in order to reduce the switching losses, the soft switching operation should be guaranteed. Therefore, it only limits that the soft switching points can be selected to inject energy to the resonant tank. To some extent, this increases the diffculties of the energy injection control.

According to the system characteristics of the direct AC-AC topology, this paper firstly analyzes the energy critical equilibrium conditions based on the energy equilibrium analysis. Furthermore, this paper gives a calculation method for the damping factor, deepth and area of the current envelope pit. On this basis, the pre-conditions of the system state variables are also proposed. Secondly, an estimation model is set up to predict the current peak before energy injection. It will be helpful for solving the control lagging problem. Thridly, this 
paper proposes an optimal control strategy to adjust the energy injection density. Finally, simulation and experiment results verify the control method.

\section{PRINCIPLE ANALYSIS AND MODELING OF THE CIRCUIT}

Fig. 1 shows the topology of the energy injection resonant $\mathrm{AC} / \mathrm{AC}$ converter.

As can be seen, the circuit consists of four MOSFET switches $S_{1}, S_{2}, S_{3}, S_{4}$, four anti-parallel diodes $D_{1}, D_{2}, D_{3}, D_{4}$ and an RLC series resonant network. The switching pair composed by $S_{1}$ and $S_{2}$ is used to control the energy injection and feedback for the resonant network. Another switching pair composed by $S_{3}$ and $S_{4}$ is used to control the bidirectional energy flow under the free-running mode. At any time, only one MOSFET switch is allowed to be switched on and the others should be switched off. Its operating principle can be divided into three running modes including energy injection, energy feedback and free-running. The oscillation current peak, the input voltage and the reference peak current are three major factors for determining the running mode, as shown in Fig.2.

In the following, the positive half-cycle of the low frequency input $V_{A C}$ is selected to analyze the three modes, and to set up models for them. In order to make the controller design easy, all of the variables are transformed to dimensionless variables. The period and the peak of the low frequency input are respectively defined as $T_{l}$ and $A_{m}$, and the low-frequency input $V_{A C}$ can be expressed as $V_{A C}=$ $A_{m} \sin \left(\omega_{1} t\right)$. The free-running period of the resonant network is defined as $T_{f}$.

In the modeling, to simplify the analysis, the state variables are given by $x=u_{p} / A_{m}$ and $y=i_{p} R / A_{m}$, the time variables are given by $\tau=t / T_{f}$ and the parameter variables are given by $\beta_{1}$ $=R /\left(L_{p} T_{f}\right)$ and $\beta_{2}=T_{f} /\left(C_{p} R\right)$. All of the parameters and variables are dimensionless.

\section{A. Energy Injection Mode}

When the resonant current peak falls below the reference value $I_{r e f}$, the energy in the resonant network will be insufficient for the load requirement. At the positive half-cycle of the resonant current $i_{p}, S_{1}$ and $D_{1}$ are turned on, the other switches are turned off, and the energy is injected into resonant network. To ensure the injection current and resonant current are in the same direction, the energy injection can only be allowed in the positive half-cycle of $i_{p}$, and the energy flow direction is shown in Fig.3.

In this mode, the resonant network works as an energy storage and as an energy supply to the load, and the state equation of the system can be expressed as:

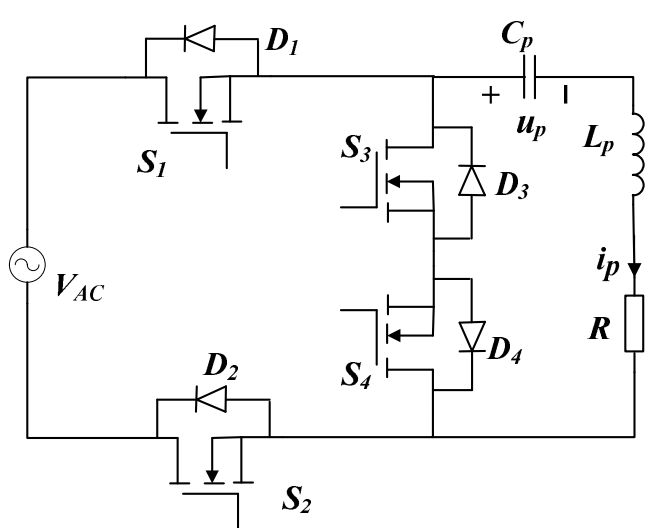

Fig. 1. Topology of the direct AC-AC converter.

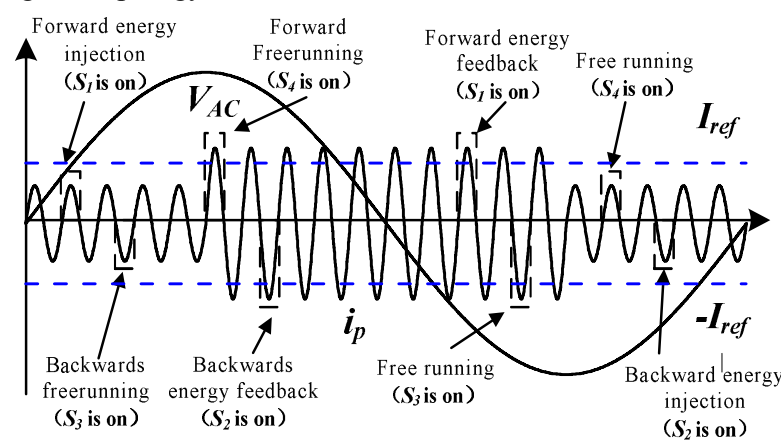

Fig. 2. Operation modes of the direct $\mathrm{AC} / \mathrm{AC}$ converter.

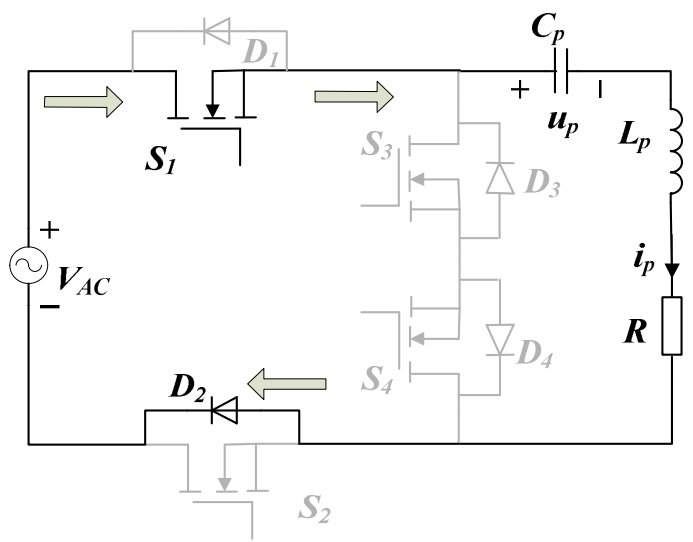

(a) Forward energy injection.

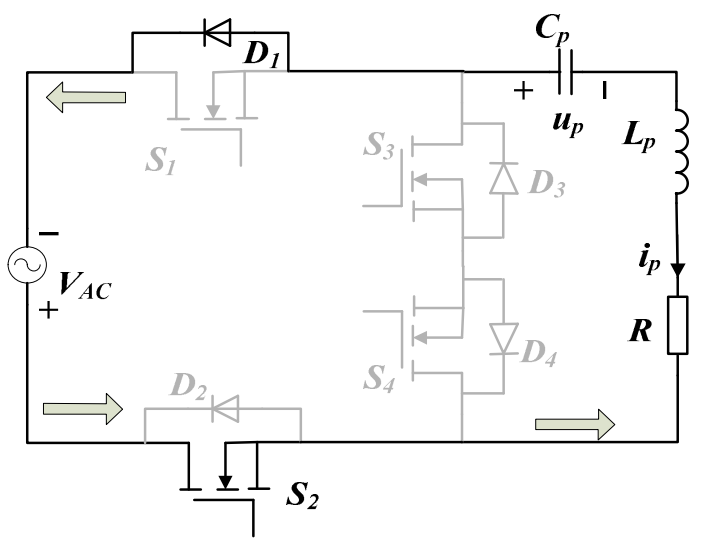

(b) Backward energy injection.

Fig. 3. Energy Injection Mode. 


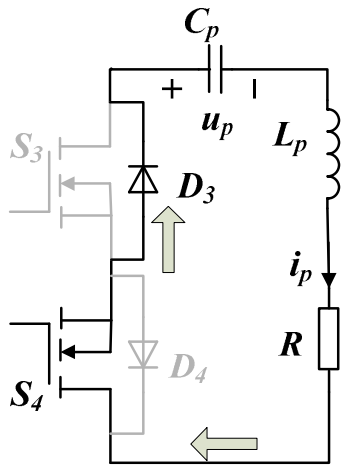

(a) Forward Freerunning.

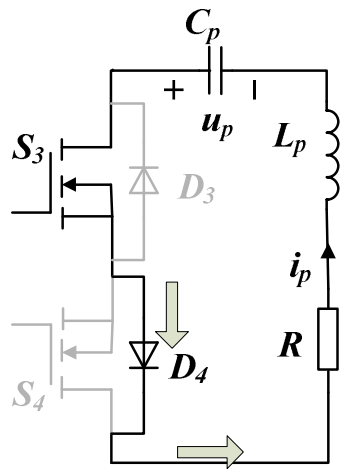

(b) Backwards freerunning.
Fig. 4. Free running mode.

$$
\left\{\begin{array}{l}
\frac{d y}{d \tau}=-\beta_{1} x-\beta_{1} y+\beta_{1} \sin (2 \pi \eta \tau) \\
\frac{d x}{d \tau}=\beta_{2} y
\end{array}\right.
$$

The initial conditions of system can be defined as $x(0)=$ $u_{0} / A_{m}$ and $y(0)=i_{p 0} R / A_{m}$. Under the initial conditions, solving the system equations can obtain the system dynamics evolution functions $x(\tau)$ and $y(\tau)$ (See Appendix).

\section{B. Free Running Mode}

The free running mode is usually implemented by the alternate conduction of $S_{3}$ and $S_{4}$. When the resonant current $i_{p}$ works in the positive half-cycle, $S_{4}$ is turned on, the current of the resonant network flows through $S_{4}$ and $D_{3}$. When $i_{p}$ works in the negative half-cycle, $S_{3}$ is turned on, and then current flows through $S_{3}$ and $D_{4}$. This complementary switching can produce the free oscillation without an external input condition. This can be shown in Fig.4.

In this mode, the state equation of the system can be expressed as:

$$
\left\{\begin{array}{l}
\frac{d y}{d \tau}=-\beta_{1} x-\beta_{1} y \\
\frac{d x}{d \tau}=\beta_{2} y
\end{array}\right.
$$

The free running mode usually operates under two conditions. The first condition is the resonant current $i_{p}$ operating in the negative half-cycle. Due to the fact that directions of the injection current and the resonant current cannot be kept consistent, the system cannot realize energy injection and it has to work in the free running mode. The other condition is that the peak of the resonant current $i_{p}$ is greater than the current reference $I_{\text {ref. }}$. Under this condition, the resonant network energy is greater than the energy needed for the load. The system should work in the free running mode to consume the excess energy and to maintain the energy balance between the resonant network and the load.

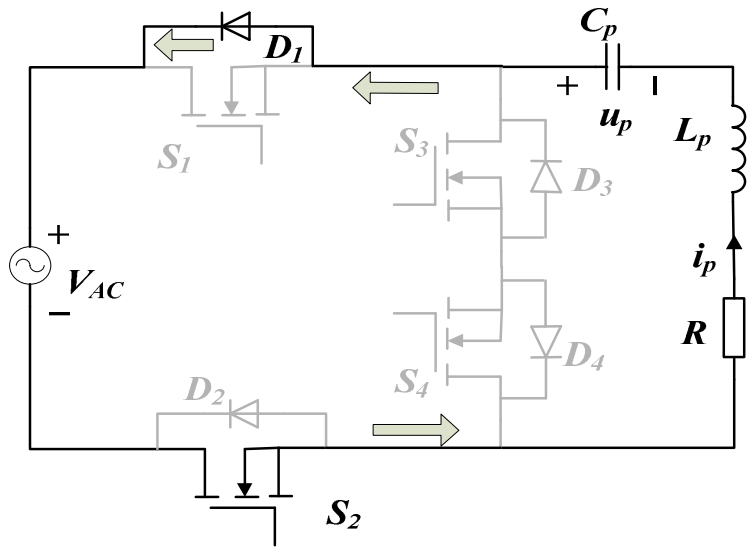

(a) Forward energy feedback.

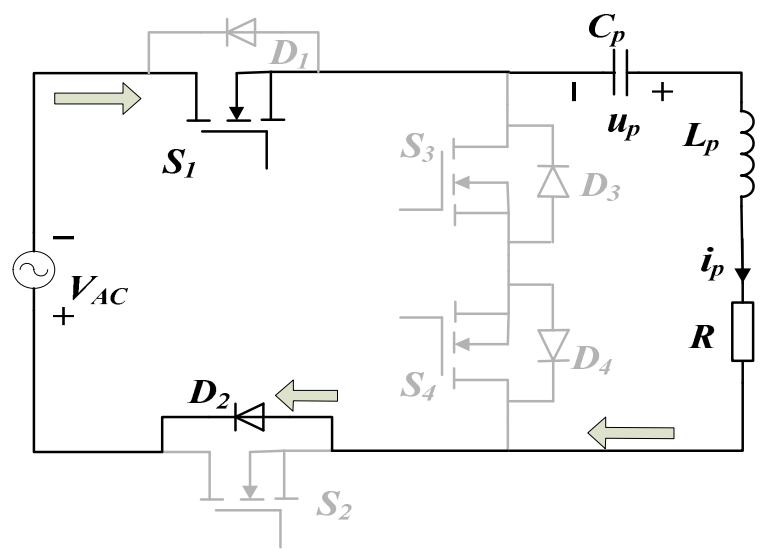

(b) Backwards energy feedback.

Fig. 5. Energy feedback mode

\section{Energy Feedback Mode}

When the resonant network energy is much larger than the energy needed for the load, the energy feedback mode should be used to feedback the excess energy into the power source. For the forward energy feedback, $S_{2}$ and $D_{1}$ are turned on, and the energy will feedback into the power source $V_{A C}$ through the resonant network. For the backward energy feedback, $S_{1}$ and $D_{2}$ are turned on. The energy feedback path can be shown in Fig.5.

In this mode, the state equation of the system is the same as the energy injection mode.

\section{ENVELOPE PIT EFFECT}

According to the analysis, the AC-AC circuit is a dissipation system and the oscillation will damp without an external energy injection. The damping factor is dependent on the parameters of the resonance RLC network. In the process of the AC-AC conversion, nearby the zero-crossing of the low frequency input, the system injection energy will be insufficient to meet the energy dissipation requirement. Thus a large pit will appear on the envelope of the resonant 
current which is named the envelope pit effect. It is found that this envelope pit is affected by the external sinusoidal input, the reference current value and the resonant circuit parameters. In order to analyze mechanism of the envelope pit effect, the energy balance equations among these parameters should be given.

The energy storage function is defined as $S\left(x\left(\tau_{0}\right), y\left(\tau_{0}\right)\right)$, the energy supply function is $P(x, y)$, and the dissipation function is $W(y)$. These functions are semi-positive definite functions. The energy balance relations can be analyzed as follows:

\section{A. Energy Injection Mode}

Assuming that the instantaneous values of the state variables in the resonant network are $x\left(\tau_{0}\right)$ and $y\left(\tau_{0}\right)$ at time $\tau_{0}$, the resonant system energy can be expressed as:

$$
S_{i}(x(\tau), y(\tau))=S\left(x\left(\tau_{0}\right), y\left(\tau_{0}\right)\right)+\int_{0}^{\tau} P(x, y)-W(y) d t
$$

\section{B. Free Running Mode}

In this mode, the resonant system energy can be expressed as:

$$
S_{r}(x(\tau), y(\tau))=S\left(x\left(\tau_{0}\right), y\left(\tau_{0}\right)\right)-\int_{0}^{\tau} W(y) d t
$$

In order to simplify the analysis, without a loss of generality, two assumptions are put forward.

Assumption 1: At the critical damping point, the system is running in the steady oscillation state, and the instantaneous value of the resonant current is $y_{r e f}=I_{r e f} R / A_{m}$.

Assumption 2: The frequency of the input AC sine wave is much smaller than the oscillation frequency of the resonance network.

At the critical damping point, according to the energy conservation theorem, the energy of the resonant network injected by the system and the dissipation energy are in balance in a cycle. Thus:

$$
S_{i}(x(\tau), y(\tau))=S_{r}(x(\tau), y(\tau))
$$

That is:

$$
\int_{0}^{1 / 2}(P(x, y)-W(y)) d \tau=\int_{1 / 2}^{1} W(y) d \tau
$$

In an energy injection oscillation system, the critical damping point is defined as $A_{b}$, its dimensionless variable is $\hat{A}=A_{b} / A_{m}$, the energy supply function can be defined as $P(x, y)=\hat{A} y$, and the dissipation function is $W(y)=y^{2}$. The equation (6) can be transformed as:

$$
\int_{0}^{1 / 2} \hat{A} y d \tau=\int_{0}^{1} y^{2} d \tau
$$

At the critical damping point, $y(\tau)=y_{\text {ref }} \sin (2 \pi \tau)$. Then the low frequency sine input $\hat{A}$ is:

$$
\hat{A}=y_{\text {ref }}(\pi / 2)
$$

To calculate the bottom value of the envelope pit, the time from the critical damping point to the bottom value and the damping envelope function should be obtained first. The fall time $t_{f}$ is defined as the time from the critical damping point to the bottom point of the envelope pit in a half-cycle, and its dimensionless variable is defined as $\tau_{f}=t_{f} / T_{f}$. This fall time is the same as the time of the low frequency input from the critical damping point to 0 . Then it can be expressed as:

$$
\sin \left(2 \pi \eta \tau_{f}\right)-\hat{A}=0
$$

where $\eta=T_{f} / T_{1}$ and $T_{1}$ is the period of the low frequency input. Combining equation (8) and (9) together the fall time can be obtained as:

$$
\tau_{f}=\arcsin \left(\pi y_{r e f} / 2\right) /(2 \pi \eta)
$$

With the triangular transformation, the solution of the state variable $y(\tau)$ in the attenuation process can be expressed as the sum of the high frequency oscillation component $\Phi(\tau)$ and the low frequency oscillation component $\Psi(\tau)$ :

$y(\tau)=\phi(\tau) \sin (h \tau-\theta)+\psi(\tau)$
where $h=\sqrt{\beta_{1}\left(\beta_{1}-4 \beta_{2}\right)} / 2, \theta=\operatorname{arctg}\left(2 h / \beta_{1}\right)$.

The envelope function of the state variables $\varphi(\tau)$ can be expressed as (where the representations of $C_{l}, H^{*}$ and $\theta^{*}$ are in Appendix):

$$
\begin{aligned}
\varphi(\tau) & =\phi(\tau)+\psi(\tau) \\
& =\frac{1}{2 \beta_{2} \sqrt{4 h^{2}+\beta_{1}^{2}}} \mathrm{C}_{1} e^{-\frac{\beta_{1} \tau}{2}}+\frac{H^{*} \pi \eta}{\beta_{2}} \cdot \cos \left(2 \pi \eta \tau+\theta^{*}\right)
\end{aligned}
$$

Therefore, the bottom value of the resonant current $y_{\text {dmax }}$ can be expressed as:

$$
y_{d \max }=\left.\varphi(\tau)\right|_{\tau=\tau_{f}}
$$

And the area of the damping section can be expressed by the function of $\tau_{f}$

$$
S_{d}\left(\tau_{f}\right)=2\left(y_{r e f} \tau_{f}-\int_{0}^{\tau_{f}} \varphi(t) d \tau\right)
$$

\section{OPTIMUM CONTROL STRATEGY FOR THE ENERGY INJECTION DENSITY}

To maintain the resonant current constant, its peak envelope should be controlled. The Bang-Bang method of envelope control proposed by [1] has the drawback of a control delay. The control actions will lag behind the error appearance. In order to solve this problem, an envelope dynamic prediction method is proposed in this paper. This method gives predictions of the coming peak points of the resonant current based on the state evolution function $y(t)$. The function under the injection and free-running modes are defined as $y_{1}(t)$ and $y_{2}(t)$, respectively (See Appendix). Since the differential equation under the feedback mode is same as the injection mode, $y_{3}(t)$ will be same as $y_{l}(t)$.

In the system, a vector $g=[x, y]^{T}$ is introduced to describe the system states. In addition, $g(\tau)$ is the state vector at time $\tau$. The predictive function $y^{*}$ at $\tau_{0}$ can be defined as:

$$
y^{*}\left(\tau^{*}, g\left(\tau_{0}\right)\right)=\left\{\begin{array}{cc}
0 & \tau^{*}<0 \\
y\left(\tau^{*}+\tau_{0}, g\left(\tau_{0}\right)\right) & \tau^{*} \geq 0
\end{array}\right.
$$


The predictive sequence of the peak points from the start of $\tau_{0}$ is defined as $\tau=\left(\tau_{1}, \tau_{2}, \cdots, \tau_{n}\right) \in R^{n}$, which satisfies:

$$
\left.\frac{d y^{*}\left(\tau^{*}, g\left(\tau_{0}\right)\right)}{d \tau^{*}}\right|_{\tau^{*}=\tau_{i}(i=1,2, \cdots n)}=0
$$

The time prediction function of the next peak point can be given by:

$$
\tau_{\text {peak }}^{*}=\min _{i}\left|\tau_{i}\right|
$$

The current prediction value of the next peak point can be expressed as:

$$
y_{\text {peak }}^{*}=y^{*}\left(\tau_{\text {peak }}^{*}\right)
$$

Based on the prediction model above, the peak time at the starting point of each mode can be predicted. It is helpful for accurate stability control.

Due to the soft switching operation requirement, the switching points of each mode should be selected at the zero-crossing points of the state variables. Therefore, optimal control of the energy injection is realized by the discrete chain decision sequence.

The decision $u_{i}$ is defined as the mapping from the start time to the end time of a certain mode, and the decision set is defined as $U=\left\{u_{1}, u_{2}, u_{3}, \cdots, u_{N}\right\}$, which corresponds to the positive energy injection, free running, backward energy injection, energy feedback and other possible operation modes, respectively. The decision $u_{i}$ can be expressed as:

$$
u_{i}\left(g(\tau), \xi_{i}\right)=\left[\begin{array}{l}
x_{i}\left(g(\tau), \xi_{i}\right) \\
y_{i}\left(g(\tau), \xi_{i}\right)
\end{array}\right], i=1,2,3 \cdots, N
$$

where the model duration $\xi_{i}$ is given by the following constraint equations:

$$
y_{i}\left(g(\tau), \xi_{i}\right)=0, i=1,2,3 \cdots, N
$$

The control strategy can be defined as a chain decision sequence consisting of $\mathrm{n}$ decisions:

$$
p_{n}=\left\{u_{k 1}\left(g\left(\tau_{0}\right), \xi_{k 1}\right), u_{k 2}\left(g\left(\tau_{1}\right), \xi_{k 2}\right), \cdots, u_{k n}\left(g\left(\tau_{n}\right), \xi_{k n}\right)\right\}
$$

where $k 1, k 2, \cdots, k n \in\{1,2, \cdots, N\}$ :

With the envelope prediction model given by equation (18), the peak point prediction sequence corresponding to the decision sequence given by equation (21) can be obtained:

$$
\left.y_{\text {peak }}^{*}\right|_{u_{k i}}=y^{*}\left(u_{k i}\left(g\left(\tau_{i}\right), \xi_{k i}\right),\left.\tau_{\text {peak }}^{*}\right|_{u_{k i}}\right)
$$

The iterative monotone non-decreasing objective function for the control can be defined as:

$$
J_{k}\left(g\left(\tau_{k}\right), p_{n}\right)=J_{k-1}\left(g\left(\tau_{k-1}\right), p_{n-1}\right)+\left|y_{\text {ref }}-y_{\text {peak }}^{*}\right|_{u_{k i}} \mid
$$

where $k \in\{1,2, \cdots n\}$.

Based on the iterative objective function, the corresponding optimal control function for the constant current envelope can be established as:

$$
J_{k}^{*}\left(g\left(\tau_{k}\right)\right)=\min _{p_{k i} \in p_{k n}\left(g\left(\tau_{k}\right)\right)} J_{k}\left(g\left(\tau_{k}\right), p_{n}\right)
$$

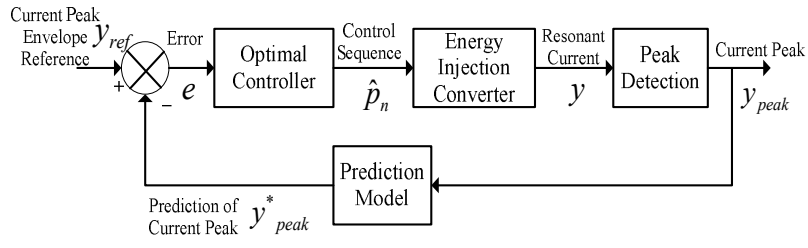

Fig. 6. Control system structure.

TABLE I

PARAMETERS OF EXPERIMENT SYSTEMS

\begin{tabular}{|c|c|}
\hline Input sine voltage peak $A_{m}$ & $20 \mathrm{~V}$ \\
Input sine current frequency $f_{0}$ & $50 \mathrm{~Hz}$ \\
Primary resonant converter $L_{P}$ & $402 \mu \mathrm{H}$ \\
Resonant current frequency $f$ & $56 \mathrm{KHz}$ \\
Primary resonant capacitor $C_{p}$ & $0.033 \mu \mathrm{F}$ \\
Reference current $I_{r e f}$ & $2.2 \mathrm{~A}$ \\
Load resistance $R$ & $0.2 \Omega$ \\
\hline
\end{tabular}

The optimal decision sequence is defined as a group decision sequence $p_{n}$ to see to it that the optimal control function reaches its minimum. That is:

$$
\hat{p}_{n}=\left\{u_{k 1^{1}}, u_{k 2^{\prime}}, \cdots, u_{k n^{\prime}}\right\}
$$

where $k 1^{\prime}, k 2^{\prime}, \cdots, k n^{\prime} \in\{1,2, \cdots, N\}$.

Based on the optimal control method above, the control system structure can be obtained, as shown in Fig.6.

In this control system structure, for the resonant current, its error between the peak envelope reference $y_{\text {ref }}$ and the predicted value of the current peak $y_{\text {peak }}^{*}$ in the next period is defined as $e$, and the control sequence $\hat{p}_{n}$ can be obtained after the optimal controller. The control sequence can decide the next operation mode of the energy injection converter to control the resonant current peak envelope so that it always varies with reference $y_{\text {ref. }}$. The optimal controller is composed by the control strategy from equations (19) to (23), and the prediction of the current peak $y_{\text {peak }}^{*}$ can be given by the prediction model (from equations (15) to (18)).

\section{SIMULATION AND EXPERIMENTAL RESULTS VERIFICATION}

To verify the control method, simulations and an experimental system were set up for verification. The system parameters are listed in Table I.

As mentioned above, due to the existence of the envelope pit effect, the stability pre-conditions should be checked in advance, according to equations (8) and (13). The critical damping point of the input sinusoidal voltage is $A_{b}=1.2 \mathrm{~V}$, the fall time of the envelope pit effect is $t_{f}=1.2 \mathrm{~ms}$, and the bottom value of the damping is $I_{d \max }=1.4 \mathrm{~A}$.

According to the control method, the system structure for the simulation and experiment can be shown in Fig. 7.

where $k \in\{1,2, \cdots n\}$. 


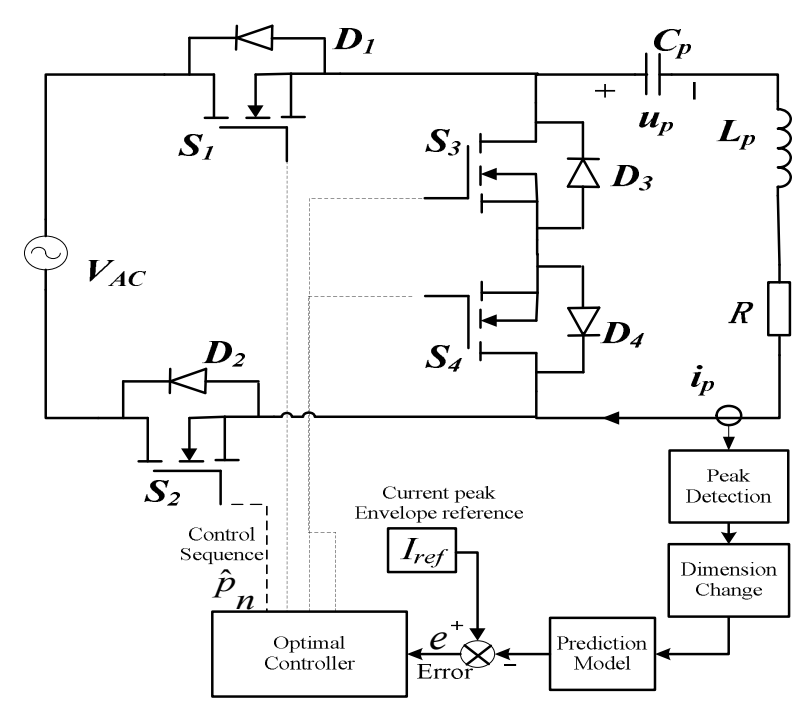

Fig. 7. Detailed system structure.
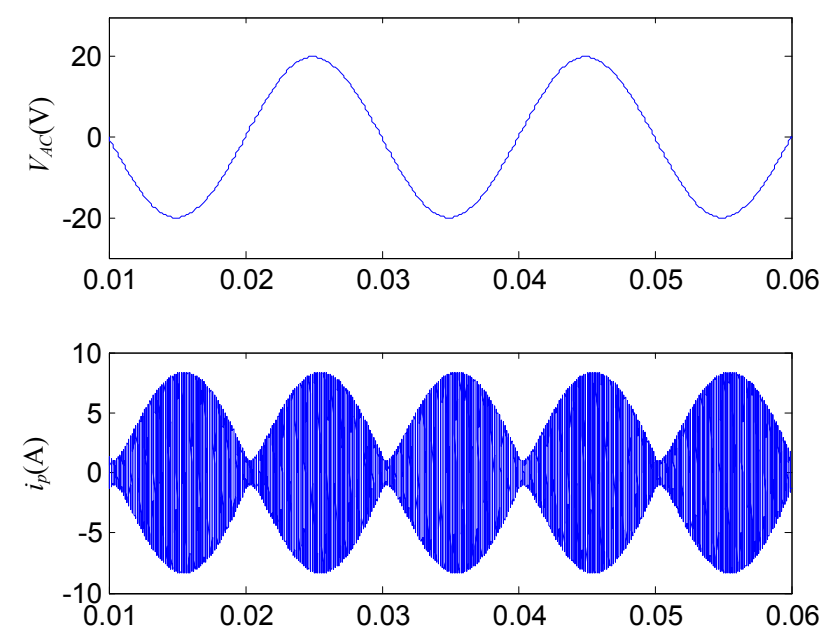

Fig. 8. Resonant current $i_{p}$ waveform of without energy injection control.

For comparison, Fig.8 shows the simulated resonant current $i_{p}$ waveform without energy injection control. The simulation time is from $0 \mathrm{~s}$ to $0.06 \mathrm{~s}$ and the maximum step size is 1 ns. The upper part in Fig. 8 is the simulated waveform of the input voltage $V_{A C}$. The lower part of this figure is the waveform of the resonant current $i_{p}$. As can be seen, the envelope of $i_{p}$ is greatly influenced by the sinusoidal voltage input. The peak value and the bottom value of the current envelope are $7 \mathrm{~A}$ and $1.2 \mathrm{~A}$, respectively.

In order to keep the resonant current envelope constant, the energy injection control method was applied. The control aim is to maintain the current envelope at a constant $2.2 \mathrm{~A}$. The simulation time is set to be from $0 \mathrm{~s}$ to $0.035 \mathrm{~s}$ and the maximum step size is 1 ns. The simulation results can be shown in Fig. 9.
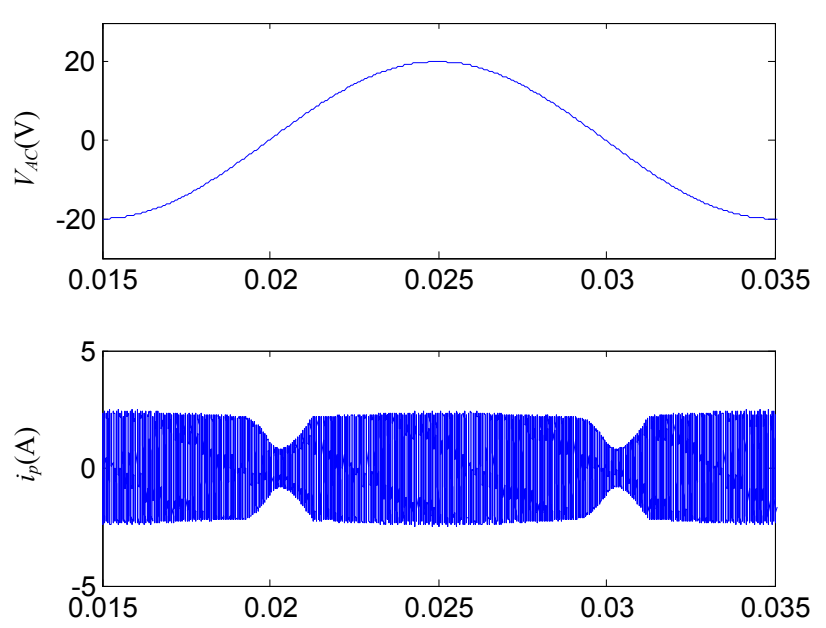

Fig. 9. Resonant current $i_{p}$ waveform of with optimal energy injection control.

As can be seen, with the energy injection control, the influence of the low frequency sinusoidal input is greatly reduced. The envelope of the resonant current $i_{p}$ is controlled at $2.2 \mathrm{~A}$. The envelope pit depth of the resonant current is 1.4 A.

To verify the simulation results, an experimental system was constructed. The experimental system uses an EP2C5T144 type FPGA as min control unit and it uses a current transformer to obtain the signal of the resonant current $i_{p}$. The peak prediction circuit consists of differential comparative circuits and the FPGA chip was utilized to implement the dimension change and the prediction of resonant current. According to the error between the predicted peak envelope and the reference, the system will produce a control sequence via the optimal control algorithm embedded in the FPPGA chip. It will also produce driving signals for the switching devices from $S_{1}$ to $S_{4}$.

For comparison, experimental results with and without the energy injection control are shown in Fig.10 and Fig.11. The measured waveforms of the input voltage $V_{A C}$ and the resonant current $i_{p}$ are shown as:

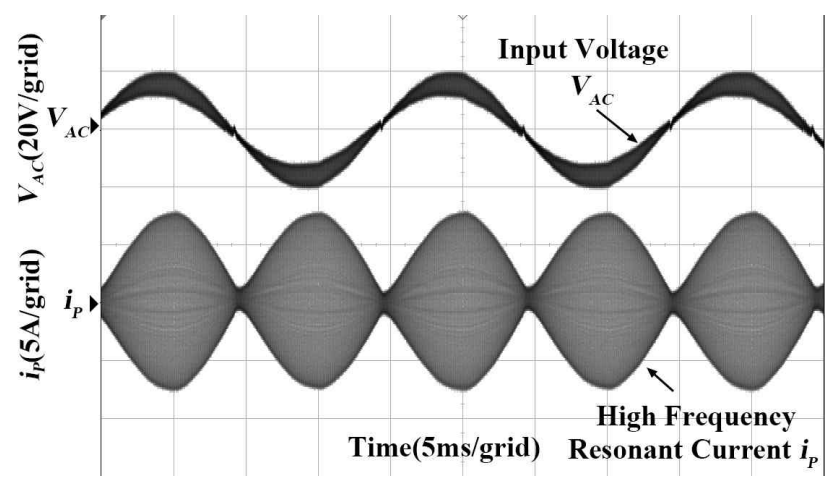

Fig. 10. Resonant current $i_{p}$ waveform of without energy injection control. 


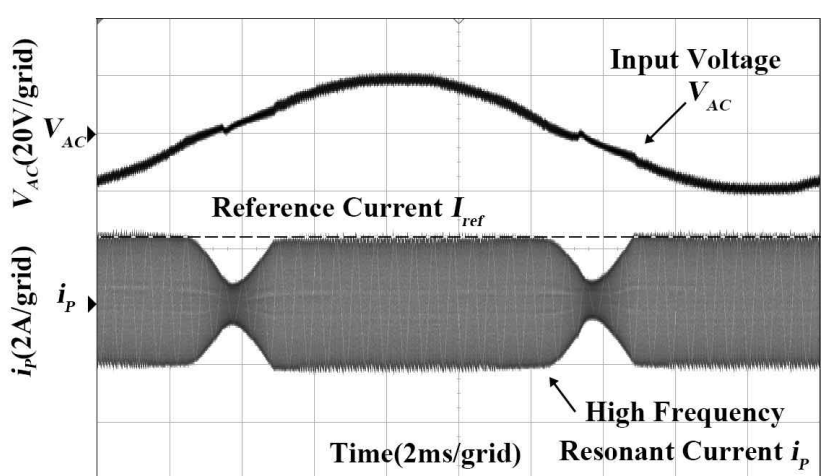

Fig. 11. Resonant current $i_{p}$ waveform of with optimal energy injection control.

Comparing Fig. 10 with Fig. 11, it can be seen that the error of the resonant current envelope can be controlled in the range of $0.2 \mathrm{~A}$ in the steady state. This verifies effectiveness of the control method. At the zero crossing point of the AC input $V_{A C}$, the envelope pit depth of the resonant current is 1.5 $\mathrm{A}$ and its fall time is $1.3 \mathrm{~ms}$. This approaches the calculated and simulated results.

\section{COMPARISON}

In order to compare the optimal control with the conventional control method, simulations and an experimental system were set up. The conventional method is a normal Bang-Bang control method. The Bang-Bang control method simply compares the resonant current with the reference signal to judge whether energy should be injected into resonant tank or not.

The simulations and the experimental system under Bang-Bang control were constructed as follows:

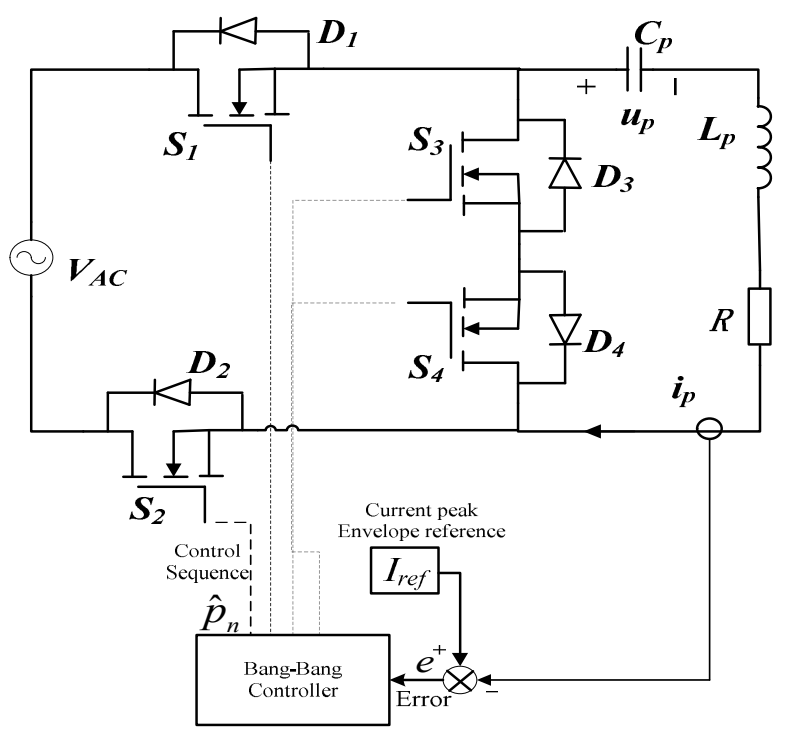

Fig. 12. Simulation and experimental system structure under conventional Bang-Bang control.
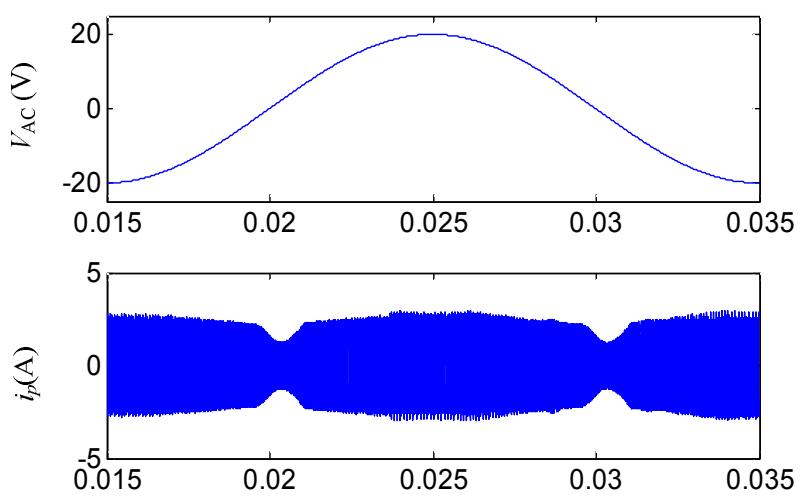

Fig. 13. Simulation waveforms of resonant current $i_{p}$ with conventional Bang-Bang control

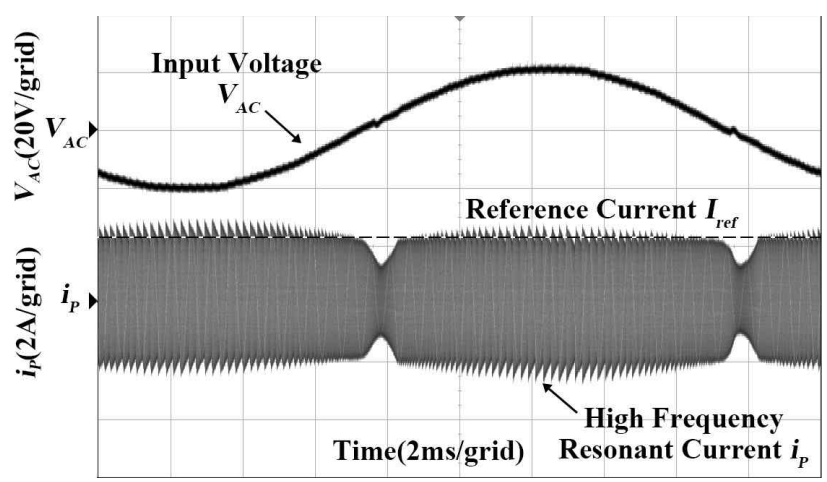

Fig.14. Experimental waveform of resonant current $i_{p}$ with conventional Bang-Bang control.

The system parameters were the same as the parameters given in Table I. Fig. 13 and Fig. 14 show the simulation and experimental results of the input voltage $V_{A C}$ and resonant current $i_{P}$ under the Bang-Bang control.

When Fig. 13 and Fig. 14 are compared with Fig.9 and Fig. 11 , it can be seen that the envelope $i_{P}$ of the resonant current cannot remain constant when the input voltage $V_{A C}$ is approaching its peak value. There are many current peaks on the envelope. This is because the injection energy and the dissipated energy cannot be keep balanced under the Bang-Bang control. Therefore, the comparison results verify the optimal control method.

\section{CONCLUSIONS}

For resonant $\mathrm{AC}-\mathrm{AC}$ converters, aiming at the constant control of the $\mathrm{AC}$ output envelope, this paper analyzes the formation mechanism of the envelope pit effect on the resonant AC current. The computing methods for the critical damping point, fall time and bottom value of the envelope pit are proposed and the stability precondition of the AC output is concluded. On the basis of the envelope prediction, this 
paper puts forward an optimal control method. With this control method, the control aim of the AC output envelope can be guaranteed and the envelope pit effect can be reduced.

\section{ACKNOWLEDGMENT}

The research work is financially supported by the research fund of International Science and Technology cooperation Plan of Chongqing (No. CSTC, 2011GGHZA40001). The plan is provided by Chongqing Science \& Technology Commission, Chongqing, China.

\section{APPENDIX}

Solution of the state equations corresponding to each mode

\section{A. Energy Injection Mode}

The state equations of the system can be expressed as:

$$
\left\{\begin{array}{l}
\frac{d y_{1}}{d \tau}=-\beta_{1} x-\beta_{1} y+\beta_{1} \sin (2 \pi \eta \tau) \\
\frac{d x_{1}}{d \tau}=\beta_{2} y
\end{array}\right.
$$

The corresponding solution is:

$$
\left\{\begin{aligned}
x_{1}(\tau)= & C_{1} e^{-\frac{\beta_{1}}{2} \tau} \sin (h \tau+\alpha)+H^{*} \sin \left(2 \pi \eta \tau+\theta^{*}\right) \\
y_{1}(\tau)= & \frac{1}{2 \beta_{2}} C_{1} e^{-\frac{\beta_{1} \tau}{2}}\left(2 h \cdot \cos (h \tau+\alpha)-\beta_{1} \sin (h \tau+\alpha)\right) \\
& +\frac{2 H^{*} \pi \eta}{\beta_{2}} \cdot \cos \left(2 \pi \eta \tau+\theta^{*}\right)
\end{aligned}\right.
$$

where:

$$
\begin{gathered}
C_{1}=\frac{x(0)-H^{*} \sin \left(\theta^{*}\right)}{\sin (\alpha)} \\
\alpha=\frac{2\left(-h \cdot x(0)+h \cdot H^{*} \sin \left(\theta^{*}\right)\right)}{4 \eta \pi H^{*} \cos \left(\theta^{*}\right)+H^{*} \beta_{1} \sin \left(\theta^{*}\right)-2 y(0)-\beta_{1} x(0)} \\
H^{*}=\sqrt{\frac{\beta_{1}^{2} \beta_{2}^{2}}{16 \pi^{4} \eta^{4}+4 \pi^{2} \eta^{2} \beta_{1}\left(\beta_{1}-2 \beta_{2}\right)+\beta_{1}^{2} \beta_{2}^{2}}} \\
\theta^{*}=\operatorname{arctg}\left(\frac{2 \pi \eta \beta_{1}}{4 \pi^{2} \eta^{2}-\beta_{1} \beta_{2}}\right)
\end{gathered}
$$

\section{B. Free Running Mode}

The state equations of the system can be expressed as:

$$
\left\{\begin{array}{l}
\frac{d y_{2}}{d \tau}=-\beta_{1} x-\beta_{1} y \\
\frac{d x_{2}}{d \tau}=\beta_{2} y
\end{array}\right.
$$

The corresponding solution is:

$$
\begin{aligned}
& \left\{\begin{array}{l}
x_{2}(\tau)=C_{1} e^{-\frac{\beta_{1}}{2} \tau} \sin (h \tau+\alpha) \\
y_{2}(\tau)=\frac{1}{2 \beta_{2}} \mathrm{C}_{1} e^{-\frac{\beta_{1} \tau}{2}}\left(2 h \cdot \cos (h \tau+\alpha)-\beta_{1} \sin (h \tau+\alpha)\right)
\end{array}\right. \\
& \text { where: } C_{1}=\frac{x(0)}{\sin (\alpha)}, \quad \alpha=\frac{2(h \cdot x(0))}{2 y(0)+\beta_{1} x(0)} .
\end{aligned}
$$

\section{REFERENCES}

[1] K. Yamada, T. Higuchi, and H. Hara, "Optimization of input and output filters in matrix converter drive system," IEEE Trans. Ind. Applicat., Vol. 127, No. 1, pp. 36-43, 2007.

[2] M. K. Nguyen, Y. G. Jung, and Y. C. Lim, "Single-phase z-source AC/AC converter with wide range output voltage operation," Journal of Power Electronics, Vol. 9, No. 5, pp. 736-747, Sep. 2009.

[3] P. Zanchetta, P. W. Wheeler., and J. C. Clare, "Control design of a three-phase matrix-converter-based AC-AC mobile utility power supply," IEEE Trans. Ind. Applicat., Vol. 55, No.1, pp. 209-217, Jan. 2008.

[4] X. J. Zheng and D. L. Chen, "Reference sinusoid circuit of grid-connected photovoltaic system based on software phase lock technique," Dianli Zidonghua Shebei / Electric Power Automation Equipment, Vol. 29, No. 6, pp. 102-105, Jun. 2009

[5] D. S. Sha, Z. Q. Guo, and K. Deng, "Parallel connected high frequency ac link inverters based on full digital control," Journal of Power Electronics, Vol. 12, No. 4, pp. 595-603, Jul. 2012.

[6] D. L. Chen and J. Liu, "The uni-polarity phase-shifted controlled voltage mode AC-AC converters with high frequency AC link," IEEE Trans. Power Electron., Vol. 21, No. 4, pp. 899-905, Jul. 2006.

[7] D. L. Chen and L. Li, "Bi-polarity phase-shifted controlled voltage mode $\mathrm{AC} / \mathrm{AC}$ converters with high frequency AC link," PESC Rec. IEEE Annu. Power Electron. Spec. Conf., pp. 677-682, 2003.

[8] H. H. Wu, J. Boys, G. A. Covic, S. Ren, and P. Hu, "An AC processing pickup for IPT systems," IEEE Energy Convers. Congr. Expo., pp. 840-846, 2009.

[9] H. L. Li, P. Hu, J. F. Gao, and X. Dai, "Development of a direct AC-AC converter based on a DSPACE platform," International Conference, Power System Technology., pp. $1-6,2006$

[10] H. L. Li, P. Hu, and G. A. Covic, "Primary current generation for a contactless power transfer system using free oscillation and energy injection control," Journal of Power Electronics, Vol. 11, No. 3, pp. 256-262, May 2011.

[11] D. Kirubakaran and S. R. Reddy, "Closed loop controlled AC-AC converter for induction heating," Journal of Power Electronics, Vol. 25, No. 2, pp. 1-5, Mar. 2009.

[12] D. Kirubakaran and S. R. Reddy, "Embedded controlled pulse converter fed induction heater," Journal of Circuits, Systems and Computers, Vol. 19, No. 3, pp. 581-595, May 2010.

[13] O. Lucia , F. Almazan, J. Acero, J. M. Burdio, and C. Carretero, "Multiple-output resonant matrix converter for multiple-inductive-load systems," Conf. Proc. IEEE Appl. Power Electron. Conf. Expo., pp. 1338-1343, 2011. 


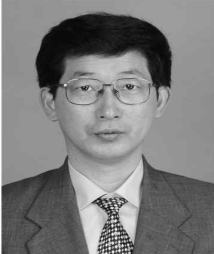

Yu-Gang Su received his B.E. and M.E. in Industry Automation and his Ph.D. in Control Theory and Control Engineering from Chongqing University, Chongqing, China, in 1985, 1993 and 2004, respectively. From 2008 to 2009 , he was a Visiting Scholar at the University of Queensland, Brisbane, Australia. He is currently working as a Professor in the College of Automation, Chongqing University. His current research interests include power electronics converters and wireless power transfer.

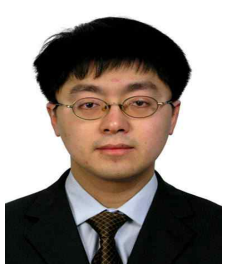

Xin Dai received his B.E. in Industrial Automation from Yuzhou University, Chongqing, China, in 2000. He received his Ph.D. in Control Theory and Control Engineering from the College of Automation, Chongqing University, Chongqing, China, in 2006. He is currently working as an Associate Professor in the College of Automation, Chongqing University. His current research interests include inductive power transfer technology and nonlinear dynamic behavior analysis of power electronics.

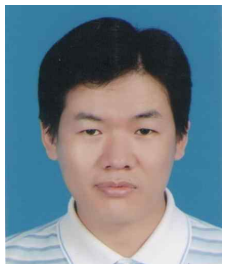

Zhi-Hui Wang received his B.E. and M.E. in Automation and his Ph.D. in Control Theory and Control Engineering from the College of Automation, Chongqing University, Chongqing, China, in 2003, 2006 and 2009, respectively. He is currently working as an Associate Professor in the College of Automation, Chongqing University. His current research interests include both fundamental investigations and practical engineering applications in efficient high-power converters and wireless power supply.

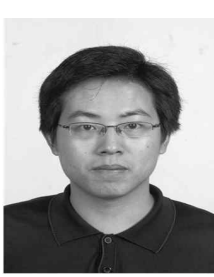

Chun-Sen Tang received his B.E. in Automation and his Ph.D. in Control Theory and Control Engineering from the College of Automation, Chongqing University, Chongqing, China, in 2004 and 2009, respectively. He was a Research Fellow in the Department of Electrical and Computer Engineering, The University of Auckland, Auckland, New Zealand, from 2007 to 2008. He is currently working as an Associate Professor in the College of Automation, Chongqing University. His current research interests include both fundamental investigations and practical engineering applications in the modeling and control of switch mode nonlinear circuits, resonant converters and wireless power transfer systems.

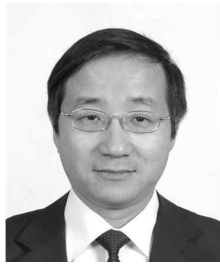

Yue Sun received his B.E. in Electrical Engineering, his M.E. in Industry Automation and his Ph.D. in Mechanical Electrical Integrated Manufacturing from Chongqing University, Chongqing, China, in 1982, 1988 and 1995, respectively. In 1997, he was a Senior Visiting Scholar in the University of Valenciennes, France. $\mathrm{He}$ is currently a Professor in the College of Automation, Chongqing University. His current research interests include automatic control, wireless power transfer and power electronics applications. 